\title{
Gestion durable d'une ressource en eaux souterraines Cas de la nappe de Beauce
}

\author{
Sustainable management of a groundwater resource \\ The case of the Beauce aquifer
}

par D. Le Coz

Direction Régionale de l'Environnement du Centre

The Beauce aquifer $\left(9000 \mathrm{~km}^{2}\right)$ is one of the main aquifers in France and plays an essential role for the rivers recharge, the drinking water supply and the ecconomic activities. The increasing pressure of agriculture, together with dry periods lead to a lack of balance and to hard tenseness. A global management of the resources with the concermed partners was set up ; the elaboration of a SAGE has been programmed.

\section{G SITUATION GÉNÉRALE}

Le complexe aquifère des calcaires de Beauce, communément appelé "nappe de Beauce" constitue une unité hydrogéologique qui s'étend sur environ $9000 \mathrm{~km}^{2}$ entre Seine et Loire. Il se trouve réparti sur deux grands bassins (SeineNormandie et Loire-Bretagne) et, deux régions (Ile de France et Centre).

Le réservoir aquifère est constitué principalement, mais non exclusivement, de calcaires empilés dans une cuvette dont le fond est d'argile imperméable. Ces calcaires ont un coefficient d'emmagasinement assez important $(10 \%)$ dans les couches supérieures, mais il doit décroître assez rapidement avec la profondeur, ce qui conduit à être prudent dans l'évaluation du volume stocké, de l'ordre de la dizaine de milliards de $\mathrm{m}^{3}$.

Dans certains secteurs, comme la forêt d'Orléans, la nappe est captive, étant surmontée d'une couche d'argile. Mais sur sa majeure partie, elle est libre et de ce fait, elle est réalimentée directement par les eaux de pluie excédentaires qui s'infiltrent du sol vers le sous-sol (le ruissellement est globalement faible). Cette infiltration qui se produit l'hiver est, en année moyenne, de l'ordre de $110 \mathrm{~mm}$, ce qui donne, pour la partie libre de la nappe, un apport moyen d'environ $900 \mathrm{mil}$ lions de $\mathrm{m}^{3} / \mathrm{an}$.

\section{E ENJEUX ET ÉVOLUTIONS RÉCENTES}

La nappe de Beauce joue un rôle de régulation très important : de l'apport hivernal, qu'elle reçoit irrégulièrement d'ailleurs, elle assure une restitution étalée dans le temps sous la forme :

- de I'alimentation des milieux aquatiques et des cours d'eau périphériques,

- des prélèvements divers liés aux activités humaines (eau potable, industrie, irrigation).

Ainsi, dans la perspective d'une gestion durable des volumes, c'est non pas au stock d'eau pris dans sa totalité que l'on s'intéressera, mais à la tranche supérieure qui entre en jeu pour les échanges ci-dessus et assure la régulation des flux.

Une description plus détaillée des différents intérêts et usages qui dépendent directement ou non de la présence de la nappe de Beauce donne la mesure des enjeux qui concernent :

- 1'alimentation des cours d'eau tributaires (notamment l'Essonne et la Juine, la Conie et donc indirectement le Loir, mais aussi la Loire), et par conséquent le soutien des étiages et la préservation des milieux aquatiques. Les volumes moyens ainsi apportés pendant la seule période d'étiage (juin à septembre), par la nappe lorsqu'elle n'est pas influencée par les prélèvements sont de l'ordre de $50 \mathrm{Mm}^{3}$ au Loir (ce 


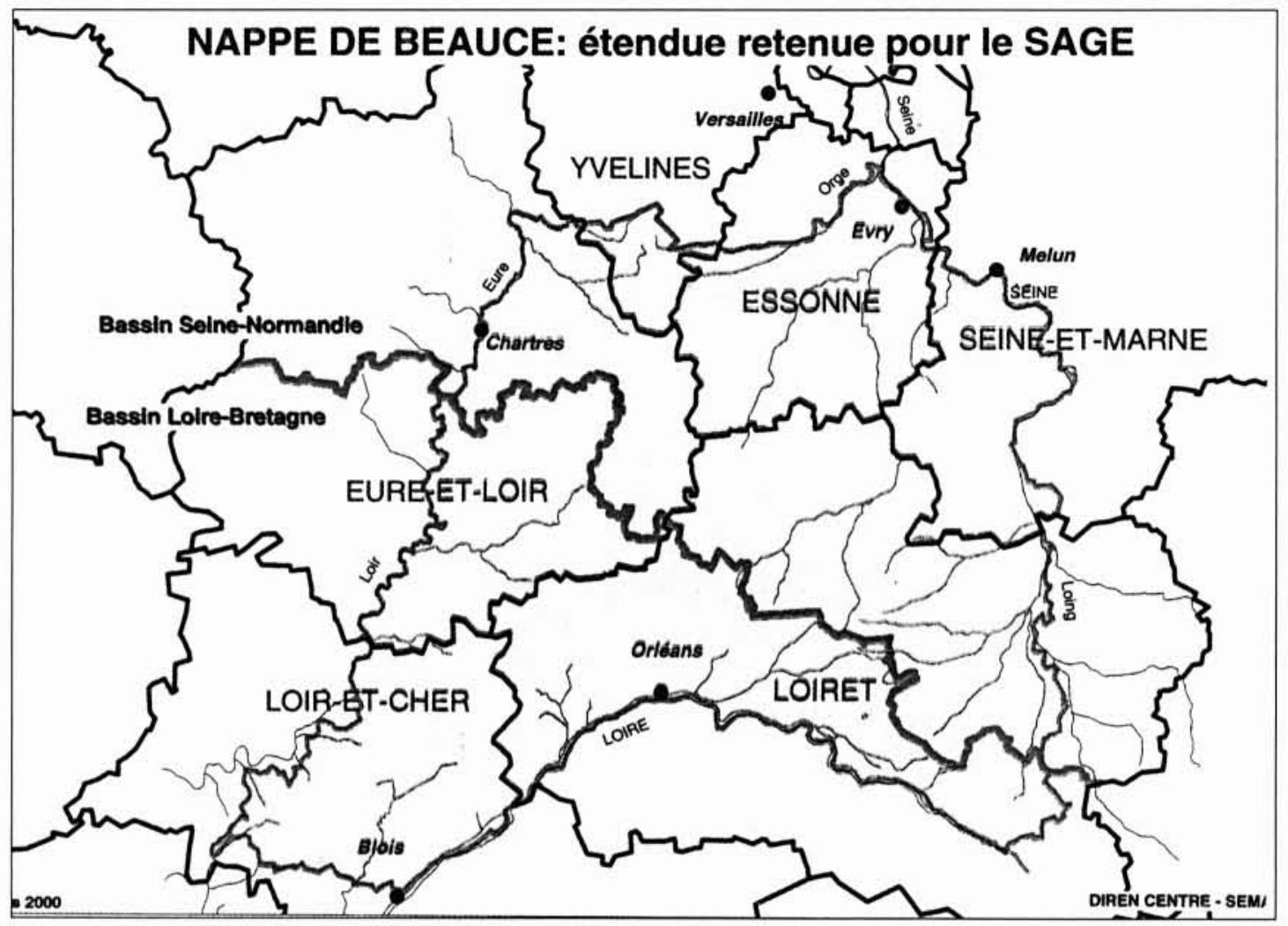

1. Carte de

la nappe

de Beauce.

qui assure un quart de l'étiage de la Maine à Angers) $100 \mathrm{Mm}^{3}$ aux affluents de la Seine, $150 \mathrm{Mm}^{3}$ à la Loire.

- l'alimentation en eau potable et de l'industrie, directement par forages pour les communes de Beauce $\left(100 \mathrm{Mm}^{3}\right)$ et indirectement à partir des cours d'eau (Loir jusqu'en Maineet-Loire),

- l'irrigation qui s'est très fortement développée passant depuis 1974 de 40000 à 250000 ha ou plus, pour un volume pouvant dépasser $300 \mathrm{Mm}^{3} /$ an et peut-être atteindre 400 ou $450 \mathrm{Mm}^{3}$.

Le volume total ainsi apporté aux milieux aquatiques et rivières ou prélevé pour les activités humaines, pendant la période estivale où l'eau est la plus précieuse, peut atteindre $600 \mathrm{Mm}^{3}$ chiffre qui mérite d'être comparé par exemple au volume stocké par un réservoir artificiel comme celui de Naussac dans le même bassin de la Loire $\left(190 \mathrm{Mm}^{3}\right)$.

Le développement des activités humaines, et en particulier d'une agriculture moderne, a introduit des modifications importantes tant quantitatives que qualitatives :

- une tendance à la baisse du niveau de la nappe, ce qui a pour effet une diminution des débits et une augmentation des assecs de certains cours d'eau exutoires, des difficultés pour l'alimentation des cressonnières (bassin de l'Essonne), ainsi que la baisse de productivité de forages situés en périphérie, - une teneur importante en nitrates dans les couches supérieures, présence de phytosanitaires, pollutions plus ponctuelles.

Les fluctuations du niveau de la nappe avaient toujours existé : fluctuations annuelles dues à l'intermittence des infiltrations, inexistantes d'avril à octobre, et qui se traduisent du fait du temps de migration de l'eau depuis le sol jusqu'à la nappe, par un maximum du niveau de la nappe vers mai et un minimum vers novembre-décembre. Egalement fluctuations interannuelles du fait de l'alternance d'années de pluviométrie hivernale plus importante ou plus faible que la moyenne.

Les observations faites par la sucrerie de Toury (45) depuis 1870 sur son puits d'alimentation constituent la série la plus longue et qui soit fiable. Elle fait ressortir des périodes de baisse continue en particulier au début du siècle : après une vingtaine d'années de déficit pluviométrique hivernal et de baisse régulière de la nappe, le niveau minimum de 106 m NGF fut observé fin 1905.

Or il a suffi en 1989-92 de 4 années de déficit pluviométrique hivernal pour ramener la nappe de Beauce à Toury d'un niveau haut $(114 \mathrm{~m})$ à celui qui avait été observé en 1905. Cela est dû à l'importance prise brutalement par l'irrigation du fait d'équipements nouveaux mais aussi de la généralisation des irrigations de printemps, sur des cultures (blé, orge, pois) qui jusqu'alors n'étaient pas irriguées.

Le cas de la Conie est une illustration extrême des effets de cette baisse sur les rivières : la Conie est un des affluents principaux du Loir, celui qui normalement contribue le plus fortement (environ $50 \%$ ) à la formation de son débit d'étiage lors des étés secs. Or cette rivière s'est trouvée complètement asséchée sur la partie aval antérieurement pérenne, les prélèvements ayant fait baisser la nappe au-dessous du niveau de son lit.

Il en a résulté évidemment une situation de fort conflit avec les riverains. Ceux-ci constitués en association ont demandé la mise en place par l'Etat de mesures de limitation des prélèvements d'irrigation qu'il n'a pas été possible d'élaborer et mettre en œuvre immédiatement eu égard à l'ampleur du problème : à titre d'indication, l'irrigation concerne plus de 3000 exploitations agricoles. 


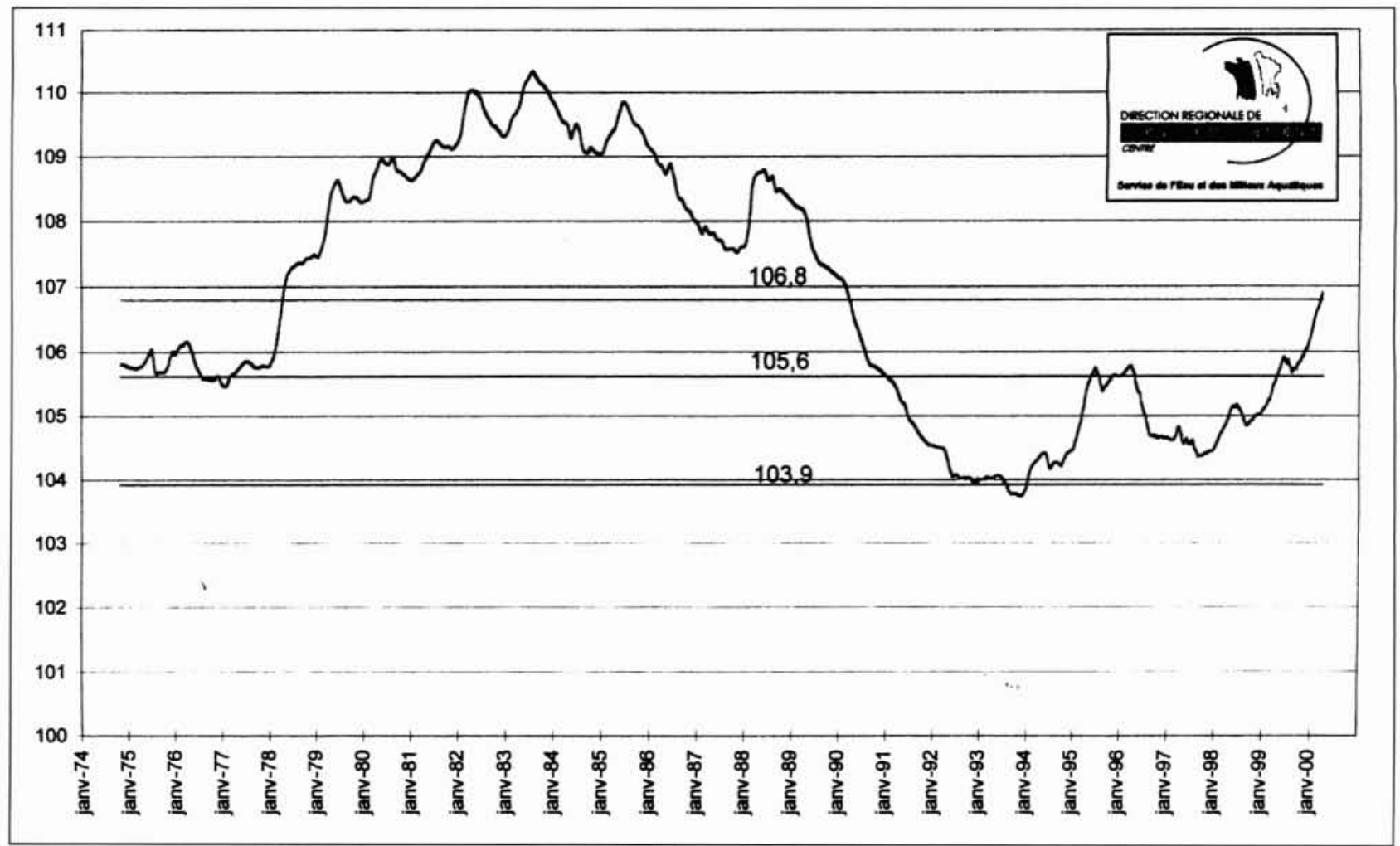

2. Indicateur du niveau de la nappe.

L'affaire a fini par être portée devant les tribunaux administratifs, ce qui valu à l'administration plusieurs annulations de ses décisions au motif qu'elles étaient insuffisantes.

Il était devenu évident qu'une gestion équilibrée et globale de la nappe de Beauce était devenue une nécessité pour préserver à la fois la ressource, les équilibres naturels, l'alimentation en eau potable et les activités économiques, conformément aux principes posés par la loi sur l'eau en son article 2 .

\section{ACTIONS ENVISAGEABLES}

Jusqu'en 1992, la puissance publique n'est aucunement intervenue pour réguler les usages ayant un impact sur la nappe de Beauce. Certes les pouvoirs de l'administration étaient très limités : en dehors des mesures toujours possibles dans le cadre des pouvoirs de police générale pour la sécurité et la salubrité, peu adaptés à la circonstance et pour lesquels les infractions sont faiblement réprimées, seule existait pour les eaux souterraines l'obligation de déclaration prévue par la loi de 1964 et un décret de février 1973, sans autre prescription technique que la pose d'un compteur et tenue d'un registre.

Encore cette réglementation était-elle bien mal respectée : grande proportion de forages non déclarés et faible équipement en compteurs. Il en résultait une très mauvaise connaissance de la situation par l'administration.

Plusieurs voies étaient envisageables pour réguler les prélèvements en particulier :

- voies financières : les agences de l'eau perçoivent effectivement des redevances sur les prélèvements mais elles sont pour l'irrigation moins fortes que pour l'eau potable et de toutes façons d'un niveau notoirement insuffisant pour influer sur les comportements. Les difficultés pour faire pas- ser, lors de l'élaboration du 7è programme en 1995, des redevances de 4 à 6 centimes du $\mathrm{m}^{3}$, obligeaient à exclure l'idée de redevances de l'ordre de $0,5 \mathrm{~F} / \mathrm{m}^{3}$ ou $1 \mathrm{~F} / \mathrm{m}^{3}$ nécessaires pour influer sur les volumes prélevés.

Par ailleurs, le marché des droits à prélever aurait pu être envisagé comme mécanisme pour optimiser l'utilisation de l'eau. Encore cela suppose-t-il qu'au départ des droits soient créés par la puissance publique (on imagine mal que le marché, par négociations entre les irrigants et les autres acteurs ayant un intérêt à la préservation de la ressource, permette à lui seul d'atteindre un équilibre dans la gestion de l'aquifère). En fait, un tel dispositif fait l'objet d'une forte réaction de rejet de la part des organisations agricoles.

- voies administratives : en posant que "l'eau fait partie du patrimoine commun de la nation" et que "sa protection, sa mise en valeur... dans le respect des équilibres naturels, sont d'intérêt général", la loi sur l'eau (art.1) légitime très fortement une intervention administrative de la puissance publique pour réguler les usages des ressources en eau.

Les objectifs d'une gestion équilibrée, visant à protéger la ressource en eau et à concilier les différents usages et exigences sont précisés à l'article 2 de la loi sur l'eau. Celle-ci définit ensuite les modes d'intervention pour parvenir à cette gestion équilibrée :

- planification au moyen de schéma directeur et de schémas d'aménagement et de gestion des eaux (SDAGE et SAGE),

- mise en cuvre de la réglementation par l'Etat (réglementation générale ; autorisation ou déclaration individuelle selon les seuils avec fixation de prescriptions techniques).

Ce sont ces voies d'action administrative qui ont été retenues et dont les différents volets ou étapes sont exposés ciaprès. 


\section{IV — NAPPE DE BEAUCE DANS LES DEUX SDAGE LOIRE-BRETAGNE ET SEINE-NORMANDIE}

Les deux SDAGE Seine-Normandie et Loire-Bretagne prennent la mesure des enjeux sur la nappe de Beauce. Ils soulignent l'importance d'une gestion équilibrée de cette ressource et dégagent à cet effet plusieurs objectifs fondamentaux :

- teneur maximale de $40 \mathrm{mg} / \mathrm{l}$ de nitrates dans la nappe et plusieurs exutoires,

- débits à maintenir dans les exutoires principaux (Essone, Juine, Conie, Mauves, Loir),

- des prescriptions en termes de niveau général de la nappe figurent dans le SDAGE Loire-Bretagne : deux seuils d'alerte et un seuil critique sont définis par rapport à un indicateur général du niveau de la nappe (moyenne pondérée des niveaux en 9 piézomètres représentatifs).

Pour ce qui est de la démarche, seul le SDAGE LoireBretagne préconise formellement la réalisation, de façon prioritaire, d'un SAGE sur la nappe de Beauce.

Le SDAGE Seine-Normandie met en avant, d'une façon générale, l'élaboration de SAGE sur des bassins versants de cours d'eau sans omettre de souligner pour les exutoires de la Beauce (Eure amont, Juine-Essonne-Ecole, Loing) la nécessité de prendre en compte les enjeux propres à cet aquifère.

Mais la réalisation éventuelle de SAGE sur les exutoires n'est en rien exclusive de celle d'un SAGE sur la nappe souterraine qui les alimente. Même à supposer que les périmètres viendraient à se superposer plus ou moins, les unités hydrologiques visées ne seraient pas les mêmes et les préconisations non plus. Se poserait simplement, tout comme pour 2 SAGE concernant l'un l'amont, l'autre l'aval d'un même cours d'eau, la question de la cohérence contre les documents. Or celle-ci est déjà en partie assurée par les objectifs de débits et de qualité fixés par le SDAGE aux points nodaux situés à l'amont des exutoires et évoqués ci-dessus.

Toujours est-il que les deux SDAGE s'accordent sur la nécessité d'une approche globale pour la gestion de la nappe de Beauce dans son ensemble et le SDAGE Seine-Normandie préconise à cet effet la création d'une commission multipartite interbassins. Une telle commission de concertation, de nature à préfigurer la commission locale de l'eau chargée d'élaborer un SAGE, a été d'ailleurs mise en place en 1997.

Concernant le périmètre proprement dit, chacun des deux SDAGE donne une indication du contour de la nappe de Beauce devant faire l'objet d'un tel effort de gestion :

- carte ${ }^{\circ} 54$ du SDAGE Seine-Normandie, annexée au chapitre "zonage pour la gestion quantitative des eaux"; la nappe de Beauce y est figurée au titre des zones de répartition des eaux à créer,

- carte $\mathrm{n}^{\circ} 8$ du SDAGE Loire-Bretagne relative aux "unités hydrographiques cohérentes", c'est-à-dire celles ne devant pas être scindées entre deux SAGE.

Au total la nappe de Beauce est définie de façon large, s'étendant sur toute la zone délimitée :

- au nord Est : en gros par la Seine,

- à l'ouest : par l'Eure amont et par le cours du Loir,

- au sud-ouest : par le bassin de la Cisse amont inclus,

- au sud : par la Loire,

- à l'est : par le bassin du Loing inclus.
Toutefois la définition reste sommaire et les SDAGE laissent d'une façon générale aux acteurs locaux le soin de préciser dans la concertation le périmètre précis des SAGE à élaborer. Le cas est pris en compte par le décret du 24 septembre 1992 qui prévoit la procédure à suivre alors (art. 2.II).

\section{$V$ - LES PREMIERES MESURES DE RÉGULATION}

Les textes d'application de la loi sur l'eau ont créé les outils de l'action de l'Etat. C'est sur leurs bases qu'ont été prises les premières mesures qui consistaient à fixer un nombre de jours de la semaine ( 1 ou 2 selon le niveau) où l'irrigation était interdite.

Les mesures de police administrative de l'eau étant de la compétence des Préfets de département, le premier objectif était d'assurer la cohérence, l'homogénéité des mesures de limitation sur l'ensemble de l'aquifère qui se trouve partagé entre 6 départements.

Il a fallu également les expliquer aux professionnels agricoles et cela s'est traduit par une "charte de l'irrigation" décrivant les modulations des mesures selon le niveau moyen de la nappe, apprécié par un indicateur qui est la moyenne pondérée des niveaux observés en 9 piézomètres représentatifs.

L'abondance du contentieux auquel donnèrent lieu les arrêtés préfectoraux ainsi pris de 1994 à 1997, tant de la part d'associations reprochant leur insuffisance que de groupements d'irrigants aux positions inverses, montrent l'acuité du conflit et des oppositions d'alors, et en particulier les fortes réticences d'une bonne partie des irrigants à admettre le principe même d'une intervention de l'Etat, dont le contenu était pourtant manifestement assez faible.

Ces mesures n'avaient de portée qu'à l'égard de ceux qui n'étaient pas suréquipés. Une bonne partie (sinon la plupart) des irrigants pouvaient autant irriguer en 6 jours qu'en 7 .

De fait, la nappe n'est guère remontée, et le volume prélevé a dû atteindre un sommet en 1996 (450 $\mathrm{Mm}^{3}$ ?).

Il était apparu évident qu'il fallait aller plus loin tant pour assurer la préservation de la nappe et des écosystèmes dépendants, que pour assurer une répartition équitable entre irrigants des prélèvements admissibles.

On s'est ainsi orienté vers un dispositif de régulation volumétrique des prélèvements en eaux d'irrigation selon des orientations à définir par un schéma d'aménagement et de gestion des eaux, propre à la nappe de Beauce et qui aborde les aspects qualitatifs et quantitatifs.

Dans la perspective de l'élaboration du SAGE, un premier dispositif d'attente a été mis en place en 1999 pour la période 1999-2001.

\section{VI — LE LANCEMENT DE L'ÉLABORATION D'UN SAGE}

Sur une unité hydrographique qui est le siège de tels enjeux et où les évolutions récentes ont fait naitre des tensions importantes entre les divers acteurs concernés, il s'agit de mettre en œuvre une gestion équilibrée de la ressource visant à concilier les différents intérêts et usages en jeu.

Or aucune action ne peut se concevoir valablement si elle ne s'inscrit pas dans une stratégie globale à long terme 
qu'il convient de définir sur l'ensemble de l'aquifère en dégageant des objectifs clairs concernant les divers aspects qualitatifs et quantitatifs (teneur en nitrates, phytosanitaires, débits dans les cours d'eau, niveaux de la nappe, priorités d'usages, modalités de répartition etc.).

Un schéma d'aménagement et de gestion des eaux (SAGE), tel que défini par la loi sur l'eau (art. 3), apparaît comme l'outil le plus adapté pour répondre à ce problème, tant par son contenu possible et ses effets en droit, que par la légitimité forte qui s'attache à son mode d'élaboration associant tous les partenaires. Le SAGE est en effet élaboré par une Commission Locale de l'Eau, réunissant les représentants des divers partenaires concernés, et présidée par un élu. Elle est constituée par le Préfet sur le périmètre qu'il définit au préalable.

Périmètre et contenu du SAGE doivent respecter les dispositions du Schéma Directeur d'Aménagement et de Gestion élaboré par le Comité de bassin. En l'occurrence, la nappe de Beauce est concernée par les deux SDAGE adoptés et publiés en 1996 pour les bassins Loire-Bretagne et Seine-Normandie et qui ont été adressés à chaque collectivité locale concernée.

Dans de telles perspectives, le principe qui doit prévaloir pour la détermination du périmètre, et qui a effectivement prévalu au cours des discussions ainsi que le prévoient les deux SDAGE, est celui qui consiste à englober l'ensemble des parties de l'aquifère qui interagissent plus ou moins étroitement, et forment un tout hydrogéologiquement cohérent, quelles que puissent être les particularités locales. En effet, si celles-ci peuvent être de nature à justifier des modulations dans le contenu des mesures à prendre, il n'est pas possible au départ d'en préjuger et il importe de n'exclure les zones concernées ni des études, ni des réflexions à mener. Il convient au contraire de les y inclure tout en prévoyant de bien prendre en compte les particularités qui leur sont propres.

Dans ces conditions, le parti a été pris, pour définir la zone de gestion globale de la nappe de Beauce, de s'appuyer sur des considérations essentiellement liées aux liaisons hydrodynamiques plus que sur les caractéristiques géologiques pures des roches formant l'aquifère.

Le périmètre a été fixé par arrêté interpréfectoral du 13 janvier 1999 pris après consultations réglementaires (notamment 700 communes).

La Commission Locale de l'Eau qui sera chargée de l'élaborer est en cours de constitution et devrait comprendre entre 60 et 70 membres.

\section{VII $\square$ LE DISPOSITIF DE GESTION VOLUMÉTRIQUE 1999-2001}

L'élaboration d'un SAGE prend plusieurs années. Or les partenaires s'accordaient sur l'idée qu'on ne pouvait pas attendre pour modifier le premier dispositif de régulation des prélèvements conçu en termes de limitation du nombre de jours d'irrigation.

Aussi a-t-il été décidé de mettre en place, dans l'attente du SAGE qui fixera le cadre et les modalités précises de gestion de la nappe, un dispositif de gestion volumétrique transitoire pour la période 1999-2001, qui aura donc vocation à être ensuite adapté pour être rendu compatible avec le SAGE.
Ce dispositif d'attente du SAGE a donné lieu en 1998 à des discussions approfondies entre les responsables professionnels agricoles et l'administration sur les 6 départements concernés.

Il a été soumis à l'approbation du groupe de travail interbassins qui a été constitué selon les recommandations des deux SDAGE et qui constitue en quelque sorte une préfiguration de la Commission Locale de l'Eau, mais avec une composition donnant une moindre place aux collectivités territoriales.

Le dispositif élaboré se présentait ainsi :

1. - Calendrier : la gestion des prélèvements par volume fixé individuellement est à mettre en œuvre sur l'ensemble de la Beauce en même temps et à partir de 1999.

2. - Une gestion pluriannuelle : pour chaque irrigant prélevant dans la nappe, un volume de référence prélevable annuellement serait à fixer pour une période de plusieurs années ( 3 ans) ; il serait assorti de la possibilité de prélever une année donnée jusqu'à $20 \%$ en plus avec un report l'année suivante :

- soit du volume éventuellement économisé par rapport à celui autorisé, dans la limite du pourcentage ci-dessus ("bonus"), — soit de celui prélevé en excédent ("malus").

\section{3. - Une adaptation en fonction du niveau de la nappe}

Le plafond globalement prélevable pour l'irrigation une année donnée résulte de l'application d'un coefficient réducteur, fonction du niveau de la nappe, à un volume de référence considéré comme globalement prélevable en situation de nappe haute.

Pour un niveau moyen tel qu'observé en 1998 et 1999 , soit un peu inférieur au seuil S2, le coefficient réducteur a été proposé à 0,8 .

Le volume de référence en situation de nappe haute a été fixé à 450 millions de $\mathrm{m}^{3} /$ an ce qui donne un plafond millions de $\mathrm{m}^{3}$ en 1999 après application du coefficient visé ci-dessus.

Cette valeur, pour 1999 correspond aux propositions de la profession agricole. Elle peut paraitre élevée. Ce plafond a été retenu pour la première année de façon à faciliter la mise en place du nouveau dispositif de gestion qui représente un changement radical par rapport aux pratiques antérieures. L'attention était attirée sur le fait que la méthodologie proposée conduirait, en l'an 2000, à ramener le coefficient de réduction de 0,8 à 0,7 ou moins si une baisse du niveau de la nappe était constatée ou si les observations et études effectuées en 1999 confirmaient les estimations des services techniques quant aux volumes dont le prélèvement en fonction du niveau de la nappe est compatible avec l'objectif d'une gestion durable.

\section{4. - Fixation des plafonds départementaux}

En utilisation comme critère la S.A.U. des irrigants, telle qu'elle ressort des données issues des déclarations des agriculteurs pour les primes compensatoires de 1997 et des données complémentaires pour la Seine-et-Marne et les Yvelines, on a pu définir les taux de répartition entre les six départements.

\section{5. - Fixation des plafonds individuels}

Il appartient à chaque Préfet de département de définir ceux-ci, dans le respect de leur plafond départemental. 
Selon l'instruction des deux Préfets coordonnateurs des bassins Loire-Bretagne et Seine-Normandie, les Préfets des 6 départements ont mis en place le dispositif au $1^{\text {er }}$ semestre 1999, sous forme d'arrêtés préfectoraux qui fixent pour chacun des 3300 irrigants le volume de référence qu'il peut prélever en moyenne lorsque la nappe est haute. Ces arrêtés prévoient ou entraînent :

- l'application d'un coefficient de réduction en fonction du niveau de la nappe,

- des modalités permettant une gestion interannuelle de son volume prélevable par chaque irrigant, pour qu'il puisse adapter ses prélèvements aux besoins qui fluctuent d'une année à l'autre en fonction des circonstances météorologiques. Ainsi la possibilité de prélever une année donnée jusqu'à 1,2 fois le volume de référence annuel est prévue ; elle est assortie d'un système de report d'une année sur l'autre du dépassement (ou inversement de l'économie) venant en déduction (ou en sus) pour le calcul du volume de référence l'année suivante.

En 1999, ce coefficient de réduction a été fixé à 0,8 , ce qui a conduit au volume global de $360 \mathrm{Mm}^{3}$.

L'application du dispositif est subordonnée au suivi par chaque irrigant des volumes prélevés (compteurs, registres et déclarations annuelles) sans quoi l'irrigation est réduite à 2 ou 3 jours par semaine.

L'équipement en compteurs est bien avancé et les premières indications donnent un prélèvement effectif en 1999 de l'ordre de $180 \mathrm{Mm}^{3}$, sans doute sous l'effet conjugué d'une gestion plus économe et d'une année plus "clémente" ayant amoindri les besoins en irrigation.

La suite de la démarche reposera sur une concertation approfondie entre tous les acteurs au sein de la Commission Locale de l'Eau chargée d'élaborer le SAGE et s'appuiera sur les différentes études engagées avec les Agences de l'eau ainsi que sur les observations des prélèvements et des niveaux de la nappe.

Il est en tout cas certain qu'une prise de conscience de la valeur patrimoniale de l'aquifère s'est opérée chez les irrigants et l'on ne peut que se réjouir de constater que la succession de deux années où la recharge hivernale a été supérieure à la moyenne a pu conduire à un net rétablissement du niveau de la nappe de Beauce, ce qui n'aurait sans doute pu se produire si les prélèvements avaient continué sans aucune modération. 